\title{
Mast cells contribute to Enterovirus 71 infection-induced pulmonary edema in neonatal mice
}

\author{
Yuefei $\operatorname{Jin}^{1} \cdot$ Chao Zhang ${ }^{1} \cdot$ Hui Wang ${ }^{2,3} \cdot$ Guangyuan Zhou $^{4} \cdot$ Xiangpeng Wang $^{2,3} \cdot$ Rongguang Zhang $^{1,2}$. \\ Shuaiyin Chen ${ }^{1}$ - Jingchao $\mathrm{Ren}^{2,4} \cdot$ Lu Chen $^{5} \cdot$ Dejian Dang ${ }^{1} \cdot$ Peng Zhang ${ }^{1}$ ' Yuanlin $\mathrm{Xi}^{1} \cdot$ Weidong $\mathrm{Wu}^{2,4}$. \\ Weiguo Zhang ${ }^{6} \cdot$ Guangcai Duan $^{1}$
}

Received: 23 January 2018 / Revised: 15 March 2018 / Accepted: 9 April 2018 / Published online: 15 May 2018

(c) United States \& Canadian Academy of Pathology 2018

\begin{abstract}
Enterovirus (EV) 71 infection has been widely acknowledged as the leading cause of severe hand, foot and mouth disease (HFMD), which may rapidly lead to fatal pulmonary edema. In this study, we established a mouse model for EV71 infection exhibiting high incidence of severe symptoms with pulmonary edema. Mast cells (MCs) accumulation, activation and allergic inflammation were found in the brains, lungs and skeletal muscle of mice after EV71 infection, especially in the lungs of mice. Levels of histamine, platelet-activating factor (PAF), interleukin (IL)-4, IL-5, IL-13, tumor necrosis factor- $\alpha$ (TNF- $\alpha$ ), nitric oxide (NO), endocrine gland-derived vascular endothelial growth factor (EG-VEGF) and noradrenaline (NA) were increased in EV71-infected lungs. In addition, EV71 infection reduced the number of pulmonary T cells, dendritic cells (DCs) and monocytes, and increased the number of lung eosinophils, Tregs and MCs. MCs number and tryptase expression in target organs or tissues posed a trend towards an increase from control to severe mice. There were positive correlations between MCs number in the brains $(r=0.701, P=0.003)$, lungs $(r=0.802, P<0.0001)$, skeletal muscles $(r=0.737$, $P=0.001)$ and mean clinical score. Thus, our results suggested that MCs contributed to the pulmonary edema during EV71 infection.
\end{abstract}

Electronic supplementary material The online version of this article (https://doi.org/10.1038/s41374-018-0075-y) contains supplementary material, which is available to authorized users.

Guangcai Duan

gcduan@zzu.edu.cn

1 Department of Epidemiology, College of Public Health, Zhengzhou University, Zhengzhou, Henan, People's Republic of China

2 Henan Collaborative Innovation Center of Molecular Diagnosis and Laboratory Medicine, Xinxiang, Henan, People's Republic of China

3 Research Center for Immunology, School of Laboratory Medicine, Xinxiang Medical University, Xinxiang, Henan, People's Republic of China

4 School of Public Health, Xinxiang Medical University, Xinxiang, Henan, People's Republic of China

5 Department of Oncology, The Third Affiliated Hospital of Xinxiang Medical University, Xinxiang, Henan, People's Republic of China

6 Department of Immunology, Duke University Medical Center, Durham, NC, United States of America

\section{Introduction}

Enterovirus (EV) genus of the Picornaviridae family containing EV71 and coxsackievirus A16 (CA16) are the main viruses responsible for the outbreaks of hand, foot and mouth disease (HFMD) worldwide. Recently, EV71 infection has been reported to pose a higher risk of severe neurological complications such as brainstem encephalitis and aseptic meningitis, and even mortality in infants and young children worldwide [1, 2]. In general, EV71 infection-caused HFMD is a typically mild, self-limiting childhood disorder with symptoms, including fever as well as rashes and blisters. However, in a handful of cases, EV71-invaded central nervous system (CNS) leads to devastating clinical outcomes such as acute flaccid paralysis, aseptic meningitis, brainstem encephalitis, encephalomyelitis or pulmonary edema [2, 3]. Among them, most of the fatal cases occurred in children aged $<3$ years was due to progressive pulmonary edema or hemorrhage [3-5]. Although the mechanism of EV71 infection-induced HFMD have been extensively studied, why EV71 infection causes pulmonary edema is yet 
unclear. Therefore, understanding the pathogenesis of fatal EV71 infection with pulmonary edema is essential to develop novel therapies.

Innate cells, such as epithelial cells, endothelial cells, tissue resident macrophages, dendritic cells (DCs) and mast cells (MCs), provide the initial lines of defense against diverse pathogens [6]. Though innate immunity plays a critical role in limiting EV71 infection [7], the role of MCs during EV71 infection remains largely unknown. MCs are generated from bone marrow-derived precursors and mature after entering tissues. MCs are best known for their role with IgE-dependent allergic disorders through high-affinity FceRI receptor [8]. MCs also express tolllike receptors (TLRs) and play an important role in pathogen surveillance [9]. Direct or indirect activation of MCs by pathogens through TLRs signaling, FceRI receptor or inflammatory factors can result in degranulation, releasing powerful mediators such as histamine, heparin, tryptase, serotonin (5-HT), accompanied by secretion of $\mathrm{T}$ helper (Th) type 2 cytokines, including interleukin (IL)-4, IL-5, IL-13 and other inflammatory factors [10-13]. Since most of MCs are located around blood vessels, the release of these mediators causes local vascular leakage and edema at the site of infection $[9,10$, 13]. Histamine also increases epithelial cell mucus production and the expression of intercellular cell adhesion molecule-1 (ICAM-1) [9, 14]. In addition, MCs-derived chemotactic factors recruit other immune cells, including eosinophils, natural killer (NK) cells, neutrophils and $\mathrm{CD} 8+\mathrm{T}$ cells to the site of infection [9].

Epidemiological studies have documented that EV71 infection-induced serum IgE and type 2 cytokines production may be associated with later risk of allergic disease such as asthma in children $[15,16]$. We speculated that MCs might be involved in the development of pulmonary disorders during EV71 infection. In the present study, we established a mouse model by infecting 3-day-old neonatal mice with EV71; upon the EV71 infection, these neonatal mice developed similar clinical symptoms. Our data suggest that MCs may play critical roles during EV71 infection.

\section{Material and methods}

\section{Ethics statement}

The number of animals and procedures used were reviewed and approved by the Life Sciences Institutional Review Board of Zhengzhou University, and the experiments were performed strictly in accordance with the Guidelines of Zhengzhou University for Animal Experiments.

\section{Virus and cells}

As described previously, EV71 strain (ZZ1350) was isolated from a nonfatal case with CNS involvement in Children's Hospital of Zhengzhou (Zhengzhou, Henan, China) [17]. Virus purification was conducted as previously described [18]. RD cells and African green monkey kidney (Vero) cells (ATCC CCL-81) were cultured in DMEM (Gibco Company, New York, USA) supplemented with $10 \%$ fetal bovine serum (FBS) (Gibco Company, New York, USA). TCID $_{50}$ were determined by plaque assay using RD cells [19]. Working stocks $\left(10^{8} \mathrm{PFU}\right.$ per ml) were stored at $-80^{\circ} \mathrm{C}$. The effect of EV71 infection on the viability of RD and Vero cells at 12, 24, $48 \mathrm{~h}$ post infection (hpi) was analyzed using a MTT assay kit (Vazyme Biotech Co. Ltd., Nanjing, China).

\section{Animal model}

Specific pathogen free BALB/c mice were obtained from the Medical Animal Center in Zhengzhou University, Henan, China, and housed in the College of Public Health of Zhengzhou University. One, 3, 5 and 7-day-old mice were intraperitoneally (i.p.) inoculated with ZZ1350 strain $\left(2 \times 10^{6} \mathrm{PFU}\right)$, and observed twice daily for 15 days for clinical symptoms and survival rates. Mock-infected mice were injected with the same volume of RD cell culture supernatants. Clinical scores were defined as follows: 0, healthy; 1 , reduced mobility; 2 , ruffled hair, hunchbacked, or ataxia; 3 , weight loss; 4 , limb weakness and; 5 , dying or death. Clinical scores $\geq 4$ was defined as severe symptoms in this study.

\section{Histopathological and immunohistochemical analysis}

Organs and tissues were harvested from euthanized mice at 7 days post infection (dpi) and immediately fixed in $4 \%$ paraformaldehyde at $4{ }^{\circ} \mathrm{C}$ for $48 \mathrm{~h}$. After fixation, paraffinembedded tissues were cut into sections of $5 \mu \mathrm{m}$ in thickness and stained with haematoxylin and eosin (H\&E). Nissl's staining was performed to observe the spinal cord injury.

For lung analysis, the lungs were washed twice with sterile PBS and then dried briefly on filter paper prior to measuring wet weight. Paraffin-embedded lung tissues were also stained with Masson's Trichrome and Periodic AcidSchiff (PAS). Alveolar space of the lung was assessed through determination of the mean linear intercepts, $L_{\mathrm{m}}$ which was calculated based on 10 randomly selected fields in each section at $\times 100$ magnification with two crossed test lines [20]. Histology scores of the brain, spinal cord and lung were quantitatively evaluated by a person blinded to 
the treatment group, as reported previously [21]. Toluidine blue stained lung slices were observed under optical microscope, and the number of toluidine blue-positive cells per $\mathrm{mm}^{2}$ was quantified in a blinded fashion using $\times 20$ magnification. The viral VP1 antigens were detected by immunohistochemical (IHC) staining as described previously [17].

\section{Immunofluorescence}

A total of $5 \times 10^{5} \mathrm{RD}$ cells were seeded in a confocal dish with $2 \mathrm{ml}$ DMEM containing 10\% FBS for $24 \mathrm{~h}$, and then were infected with 1 MOI EV71. Infected RD cells were collected at $24 \mathrm{hpi}$, and washed twice with $1 \times$ PBS. Cells were then stained with rabbit anti-EV71 VP1 polyclonal antibody (GeneTex, Inc, San Antonio, USA 1:500 dilution), followed by incubation with $\mathrm{Cy} 3$-conjugated goat antirabbit IgG (Beyotime Biotech Inc., Jiangsu, China) at 1:500 dilution.

Paraffin-embedded tissues of $5 \mu \mathrm{m}$ in thickness were dewaxed and washed three times with $1 \times$ PBS. Before being permeabilized with $1 \times$ PBS containing $0.5 \%$ Triton $\mathrm{X}-100$, the sections were blocked for $30 \mathrm{~min}$ with goat serum at room temperature, incubated with rabbit antimouse tryptase monoclonal antibody (Abcam, USA 1:1000 dilution), and then incubated with Cy3-conjugated goat anti-rabbit secondary antibodies at a concentration of 1:500 at $37^{\circ} \mathrm{C}$ for $30 \mathrm{~min}$. After staining with DAPI for $5 \mathrm{~min}$, the images were captured using Leica TCS-SP8 confocal microscopy (Leica Microsystem, Wetzlar, Germany. Positive area of tryptase expression was identified as the red staining in the cytoplasm and evaluated by Image-Pro Plus 6.0 software.

\section{Antibodies and flow cytometry analysis}

Approximately $100 \mu \mathrm{l}$ peripheral blood was collected from mice. After lysis of red blood cells, the cells were stained with different antibodies. Lung cells extraction and staining were performed as described previously [22]. We first cut the lungs in $15 \mathrm{ml}$ tubes using small scissors. After cutting, we added cold RPMI 1640 ( $1 \mathrm{ml} /$ pair of lungs), then placed the tubes on ice. Once all lungs were cut, pre-warmed $\left(37^{\circ}\right.$ C) $2 \times$ digestion medium was added to each tube $(1 \mathrm{ml} /$ pair of lungs). Next, the tubes were placed in a warm water bath $\left(37^{\circ} \mathrm{C}\right)$ and incubated under shaking for $45 \mathrm{~min}$, followed by adding $10 \mathrm{ml}$ cold MACS buffer was added to each tube. After filtering with a $100 \mu \mathrm{M}$ cell strainer, osmotic lysis buffer ( $1 \mathrm{ml} /$ pair of lungs) was added to the tubes and then incubated at room temperature for $2 \mathrm{~min}$. Lastly, the cells were spun down and resuspended in MACS buffer. Antibodies: CD45 (APC-eFlour780), CD3e (Alexa Fluor700), CD5 (eFluor450), CD4 (PE), CD8a (BB515),
CD25 (PE-Cy5.5), CD69 (PE-Cyanine7), CD44 (BV605), CD62L (APC), MHCII (FITC), Ly6G (Alexa Fluor700), NK1.1 (BV421), CD19 (PE-Cy5.5), CD11c (PE), CD11b (APC), CD5 (PE-Cy7), Foxp3 (PE) (eBioscience, Ltd., UK), CD4 (Biotin) (BD Pharmingen, San Diego, CA, USA), and CD117 (APC) (BioLegend, Inc, San Diego, CA, USA).

\section{Western blotting}

Total protein from RD cells after EV71 infection at $24 \mathrm{hpi}$ was extracted with a protein extraction kit (CWbio Company Ltd., Beijing, China) according to the manufacturer's instruction. For western blotting analysis, samples were separated by SDS-PAGE and transferred to nitrocellulose membranes. After incubation with primary antibodies, nitrocellulose membranes were washed three times and incubated with anti-rabbit secondary antibodies. Afterwards, the membranes were again washed three times, and developed with an ECL enhanced Chemiluminescence Kit (Vazyme Biotech, China).

\section{Microarray analysis of lung inflammatory cytokines}

Inflammatory cytokines in lung lysates were analyzed with a Quantibody ${ }^{\oplus}$ Mouse Inflammation Array I kit (RayBiotech, Inc. Norcross, GA; Cat. No. QAM-INF-1) by Sample Testing Services of RayBiotech, Inc.

\section{Analysis of tissue lysates}

The brains, lungs and skeletal muscles from mice were weighed and quickly lyzed in a cold isolation buffer [23] (1 $\mathrm{ml}$ per $100 \mathrm{mg}$ tissues). The lysates were spun at $800 \times g$ and $4{ }^{\circ} \mathrm{C}$ for 10 mins. The supernatants from the first centrifugation were extracted and further centrifuged at $12000 \times g$ and $4{ }^{\circ} \mathrm{C}$ for 15 mins,. The final supernatants were collected and used for the ELISA assays. Levels of histamine, PAF, IL-4, IL-5, IL-13, tumor necrosis factor- $\alpha$ (TNF- $\alpha$ ), endocrine gland-derived vascular endothelial growth factor (EG-VEGF) and noradrenaline (NA) were evaluated using ELISA kits (Cusabio Biotech Company Ltd., Wuhan, China). NO level was measured by the Griess method, as instructed by the manufacturer of the NO assay kit (Beyotime Biotech Inc., Jiangsu, China).

\section{Statistical analysis}

All experiments were repeated at least three times. Data are presented as mean \pm SEM. SPSS21.0 (IBM, Chicago, IL, USA) was used for statistical analysis. Data comparison was carried out by two-tailed Student's $t$ test. Clinical scores of experimental mice were analyzed by one-way 
A

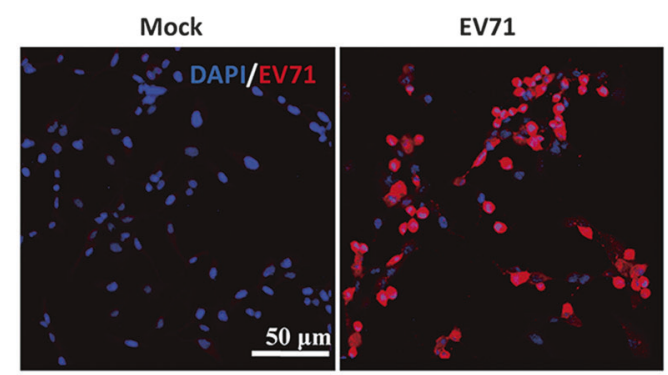

B

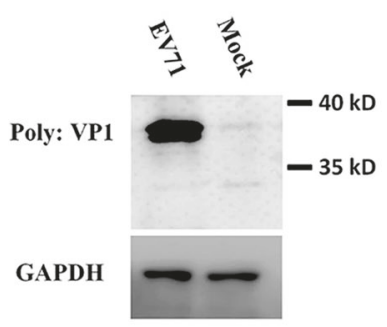

E

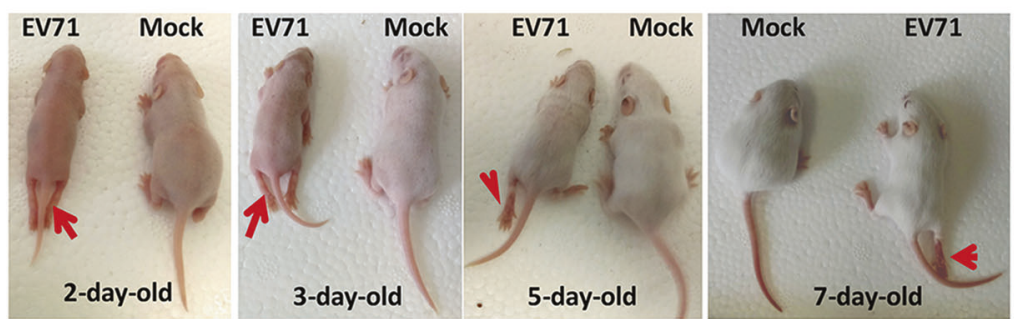

C

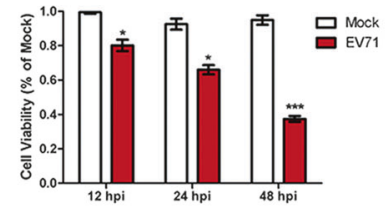

D

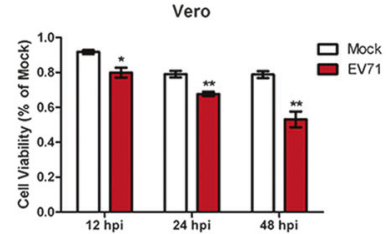

F

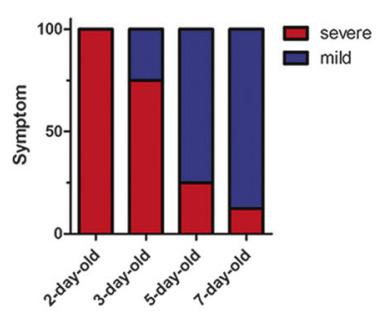

G

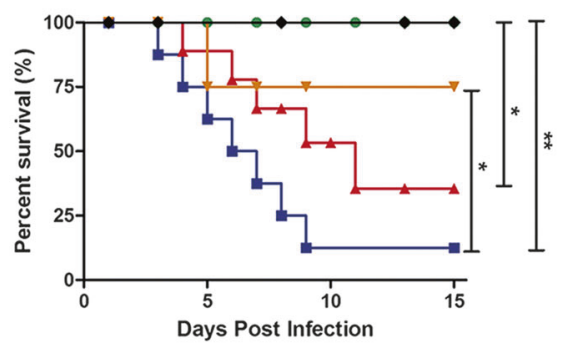

I

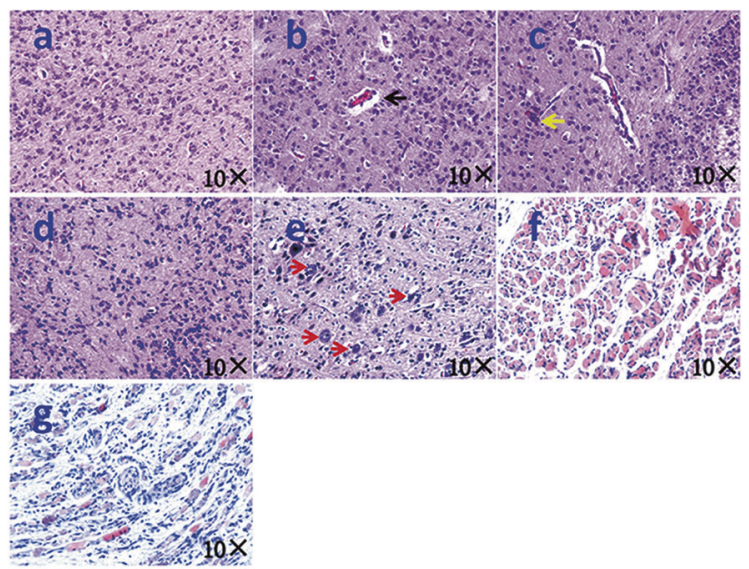

H

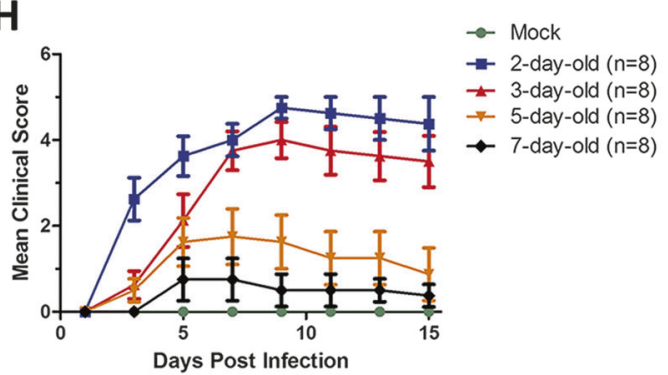

J
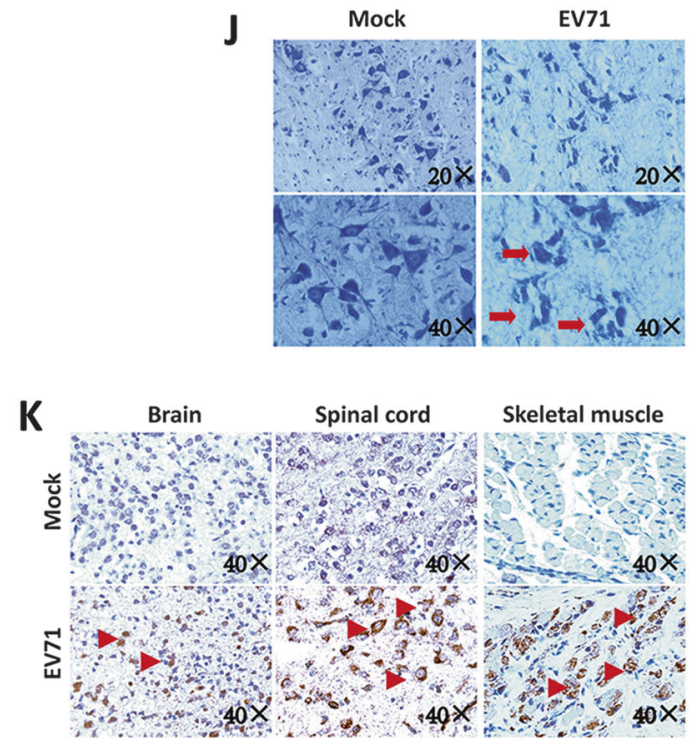

ANOVA. Survival rates of infected mice were analyzed with Log-rank test. Correlation analysis was evaluated using the Pearson's correlation test. All graphs were prepared using GraphPad Prism 5.0 (GraphPad software Inc., San Diego, CA, USA). A two-tailed $P$ value $<0.05$ was considered statistically significant. 
Fig. 1 EV71 (ZZ1350 strain) infection induced severe symptoms in neonatal mice. EV71 strain used in this study was identified by detecting VP1 expression with immunofluorescence $(\mathrm{bar}=50 \mu \mathrm{m})(\mathbf{a})$ and western blotting (b). Cytotoxicity of EV71 strain was measured using MTT assay in RD cells (c) and Vero cells (d). One-day-old, 3day-old, 5-day-old and 7-day-old mice (e) were i.p. inoculated with ZZ1350 strain $\left(2 \times 10^{6} \mathrm{PFU}\right)$, and observed twice daily for 15 days for clinical symptoms (f). Survival rate (g) and mean clinical score (h) were recorded twice daily for 15 days. To measure the histopathological alterations in target organs or tissues after EV71 infection, 3-dayold mice were inoculated with EV71 strain and were killed at 7 dpi. $\mathrm{H} \& \mathrm{E}$ stained brain tissues with amplification $(\times 10)$ from infected mice (i-b, i-c) exhibited perivascular cuffing (indicated by black arrow) and glial nodules (indicated by yellow arrow), compared to control brain (i-a). Neuronal degeneration (indicated by red arrows) was shown in H\&E stained spinal cord from infected mice with amplification $(\times 10)$ (i-e), which was not found in control mice (i-d). H\&E stained skeletal muscle with amplification $(\times 10)$ from infected mice $(\mathbf{i}-\mathrm{g})$ exhibited inflammatory cell infiltration, compared to control (i-f). j Nissl's staining of spinal cord (red arrows represent impaired neurons). Slices of brain, spinal cord, skeletal muscle of mice were stained with antiEV71 VP1 antibody (k). The red triangles indicated the positive staining (brown) and pictures were captured using a light microscope with amplification $(\times 40)$. The data are expressed as means \pm SEM. $* P$ $<0.05$, vs. control $(n=3) ; * * P<0.01$, vs. control $(n=3)$

\section{Results}

\section{EV71 (ZZ1350 strain) infection induced severe symptoms in neonatal mice}

To establish an animal model, neonatal mice of different ages were inoculated with EV71 strain (ZZ1350, C4 subtype) isolated from a nonfatal case with CNS lesions. As shown in Fig. 1a, b, this strain was able to infect RD cells. The VP1 protein of EV71 was detected in RD cells at 24 hpi by immunofluorescence staining and Western blotting. Viral infection reduced the viability of RD cells (Fig. 1c) and Vero cells (Fig. 1d) at 12, 24, 48 hpi, indicating that ZZ1350 strain was high cytotoxic in vitro. Compared to mock-infected mice, EV71-infected mice with different ages (Fig. 1e) presented with severe signs, including reduced mobility, ataxia, weight loss, spastic limb paresis and/or paralysis (fore-limbs, hind-limbs, or both) or death. As shown in Fig. 1f, the percentages of 2, 3, 5, 7-dayold mice with severe symptoms after EV71 infection were $100,75,25$ and $12.5 \%$, respectively, while the survival rates (Fig. 1g) over the 15-day observation period were $12.5 \%$, $37.5,87.5$ and $100 \%$, respectively. As shown in Fig. 1h, mean clinical scores of 2- and 3-day-old mice were higher than that in 5- and 7-day-old mice. Such results suggested that 3-day-old mice model with higher incidence of severe symptoms, which could better recapitulate the EV71 infection in humans.

To examine the histopathological alterations in different tissues after EV71 infection, we inoculated 3-day-old mice with ZZ1350 strain by i.p. and different tissues were collected at 7 dpi. As shown in Fig. 1i, vascular cuff (black arrow) and glial nodules (yellow arrow), neuronal degeneration (red arrows), necrotizing myositis with muscle fibers rupture and inflammatory cells infiltration exhibited in brain (Fig. 1i-a to c), spinal cord (Fig. 1i-d to e) and skeletal muscle (Fig. 1i-f to g), respectively. Using Nissl's staining (Fig. 1j), we noted that neuronal cells in the spinal cord of infected mice were impaired and that tissues exhibited dissolved and necrotic state.

Next, we detected the presence of virus by Immunohistochemical (IHC) staining using antibody against EV71 VP1. As shown in Fig. 1k, positive staining of VP1 (brown) was detected in cells of the brains, spinal cord and skeletal muscle sections from mice infected with EV71.

Taken together, these data indicated that ZZ1350 strain could infect different tissues in neonatal mice and cause severe symptoms linked to damaged CNS.

\section{EV71 infection caused pulmonary edema and release of inflammatory cytokines}

As pulmonary disorders are always associated with the mortality of severe HFMD [3-5], in the study we verify if this is the case in mouse model. As shown in Fig. 2a, congestive lungs was found in EV71-infected mice and the ratio of wet lung weight/body weight in EV71-infected mice was significantly elevated. The levels of $L_{\mathrm{m}}$ (Fig. 2b), EG-VEGF (Fig. 2c) and NA (Fig. 2d), which are known as the indicators of pulmonary edema, [24-26] were all increased significantly compared with the control groups. As shown in Fig. 2e, comparison of the lung tissue sections of mock and EV71-infected lungs showed that EV71 infection induced focal haemorrhage, alveolar enlargement, mucus production and erythrocyte-filled fluid in the alveolar spaces by H\&E, Masson's Trichrome and PAS staining, which were supported by their respective histology scores. These results indicated that EV71 infection could cause pulmonary disorders in neonatal mice.

To determine the involvement of inflammatory cytokines in EV71-induced pulmonary edema, the expression of inflammatory cytokines in lung lysates were measured by a Quantibody ${ }^{\oplus}$ Mouse Inflammation Array I kit. The name and location of inflammatory cytokines was listed in Fig. 2g. Fig. $2 \mathrm{f}$ showed the intensity of inflammatory cytokines expression in lung lysates from mock- and EV71-infected mice. Relative quantitative expression levels of inflammatory cytokines (Fig. 2h) showed that increased expression levels of eotaxin-1, eotaxin-2, intercellular adhesion molecule (ICAM-1), IL-7, IL-13, IL-17 were observed in lung lysates of EV71-infected mice in comparison with controls, while G-CSF, IFN- $\gamma$, IL-10 were decreased.

Taken together, our results suggested that EV71 infection caused pulmonary edema, which involved multiple 
A
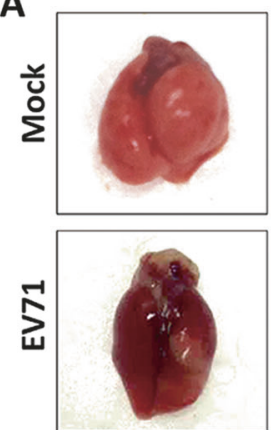

$\mathbf{F}$
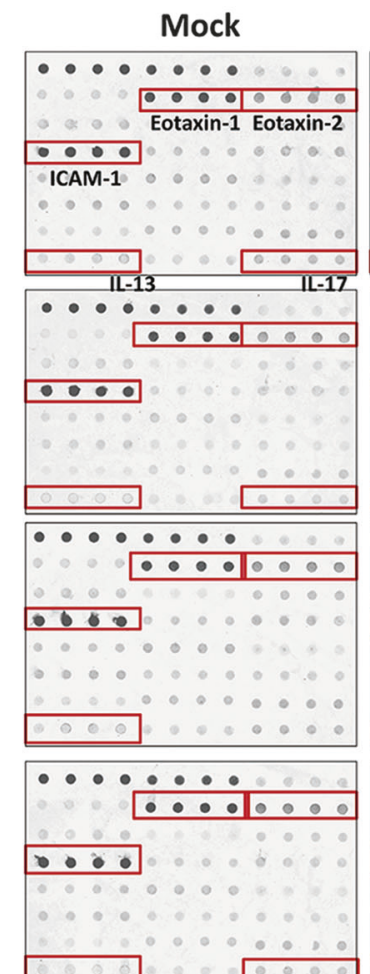

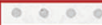

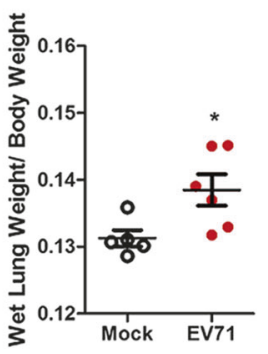

EV71

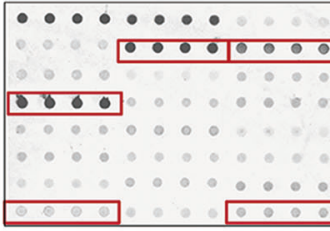

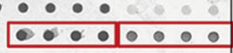
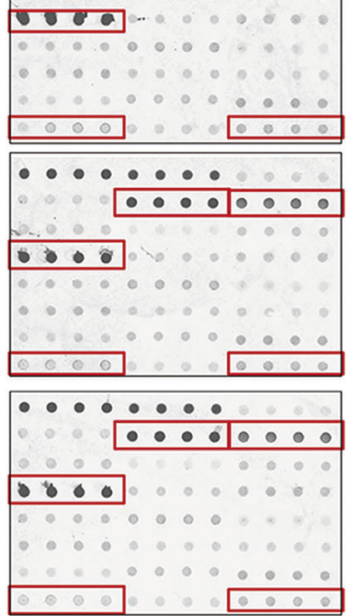

$\mathbf{G}$

\begin{tabular}{|c|c|c|c|c|c|c|c|c|c|c|c|c|}
\hline \multicolumn{13}{|c|}{ Each antibody is printed in quadruplicate horizontally } \\
\hline & 1 & 2 & 3 & 4 & 1 & 2 & 3 & 4 & 1 & 2 & 3 & 4 \\
\hline A & \multicolumn{4}{|c|}{ POS1 } & \multicolumn{4}{|c|}{ POS2 } & \multicolumn{4}{|c|}{ BLC (CXCL13) } \\
\hline$B$ & \multicolumn{4}{|c|}{ CD30 Ligand } & \multicolumn{4}{|c|}{ Eotaxin-1 (CCL11) } & \multicolumn{4}{|c|}{ Eotaxin-2 (MPIF-2) } \\
\hline C & \multicolumn{4}{|c|}{ Fas Ligand } & \multicolumn{4}{|c|}{ GCSF } & \multicolumn{4}{|c|}{ GM-CSF } \\
\hline D & \multicolumn{4}{|c|}{ ICAM-1 (CD54) } & \multicolumn{4}{|c|}{ IFN-gamma } & \multicolumn{4}{|c|}{ IL-1 alpha } \\
\hline$E$ & \multicolumn{4}{|c|}{ IL-1 beta } & \multicolumn{4}{|c|}{ IL-2 } & \multicolumn{4}{|c|}{$\mathrm{IL}-3$} \\
\hline$F$ & \multicolumn{4}{|c|}{ IL-4 } & \multicolumn{4}{|c|}{ IL-5 } & \multicolumn{4}{|c|}{ IL-6 } \\
\hline G & \multicolumn{4}{|c|}{ IL-7 } & \multicolumn{4}{|c|}{ IL-10 } & \multicolumn{4}{|c|}{ IL-12 p70 } \\
\hline $\mathrm{H}$ & \multicolumn{4}{|c|}{ IL-13 } & \multicolumn{4}{|c|}{ IL-15 } & \multicolumn{4}{|c|}{ IL-17A } \\
\hline
\end{tabular}

Fig. 2 EV71 infection caused pulmonary edema and release of inflammatory cytokines. Three-day-old mice were inoculated with ZZ1350 strain or same volume of RD cell culture supernatants and lung tissues were harvested at $7 \mathrm{dpi}$. a Representative image of mock and EV71-infected lungs, and ratio of wet lung weight/body weight from mice with EV71 infection $(n=6)$ and controls $(n=5) . L_{\mathrm{m}}$, EGVEGF and NA are known as the indicators of pulmonary edema. $L_{\mathrm{m}}$ (b) of mice lungs ( $n=5$ for mock and $n=6$ for EV71) was calculated based on 10 randomly selected fields in each section at $\times 10$ magnification with two crossed test lines. Levels of EG-VEGF (c) and NA (d) in mice lungs ( $n=5$ for mock and $n=6$ for EV71) were detected
B
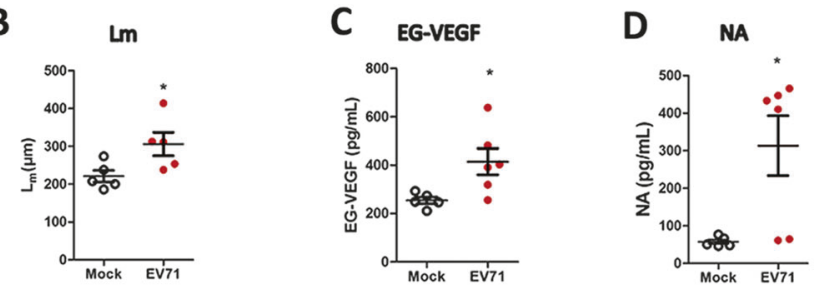

E
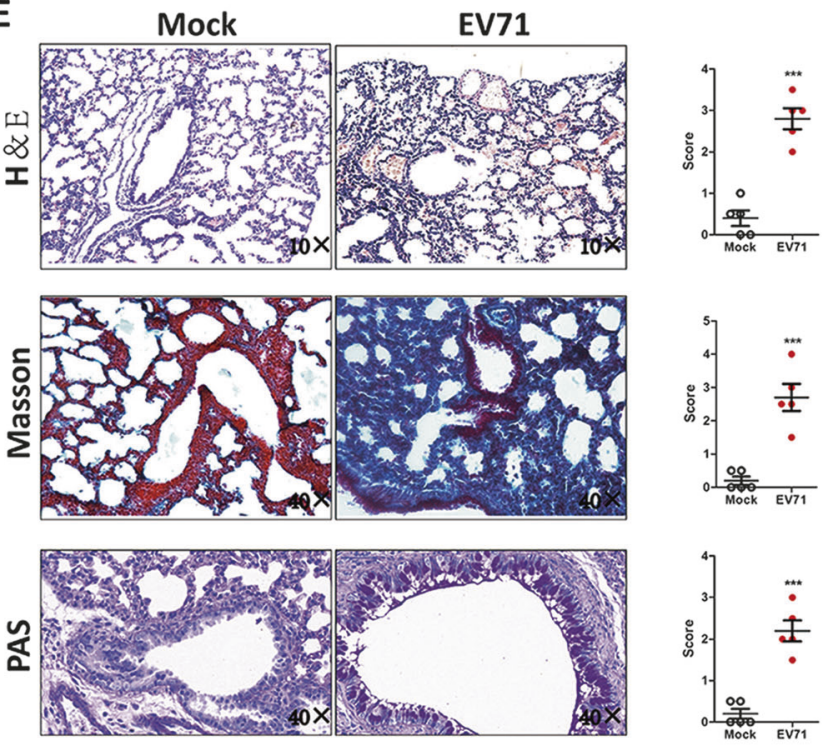

H

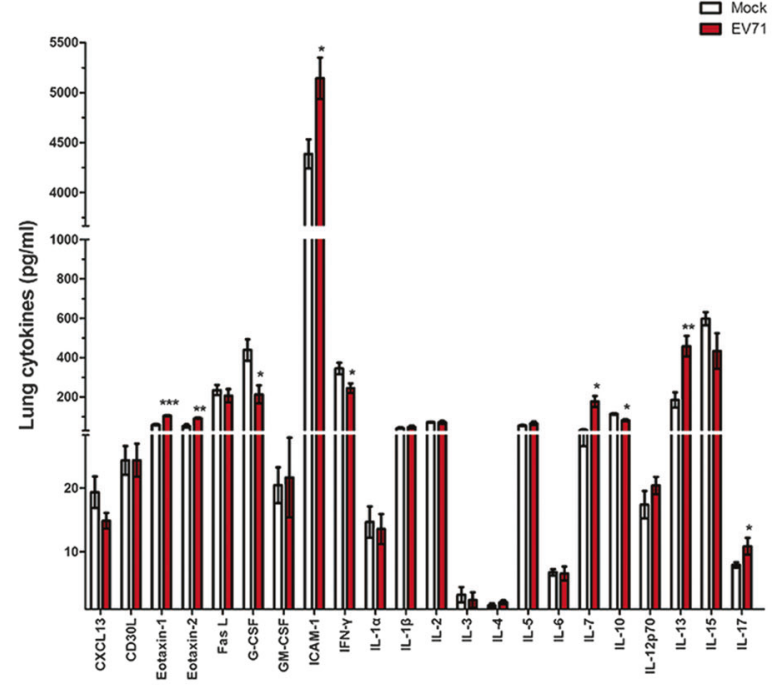

using ELISA kits. e H\&E, Masson's Trichrome and PAS stained lungs from mock $(n=5)$ and EV71 infection $(n=5)$, which was evaluated by histology score. f Inflammatory cytokines in lung lysates from mock $(n=4)$ and EV71-infected mice $(n=4)$ were measured by a Quantibody Mouse Inflammation Array I kit. g Intensity of inflammatory cytokines expression in lung lysates from mock and EV71infected mice. $\mathbf{g}$ The name and location of inflammatory cytokines list. h Relative quantitative expression levels of inflammatory cytokines in lung lysates from mock- and EV71-infected mice. The data are expressed as means \pm SEM. $* P<0.05$, vs. Mock; $* * P<0.01$, vs. Mock; $* * * P<0.001$, vs. Mock 
A Brain Lung
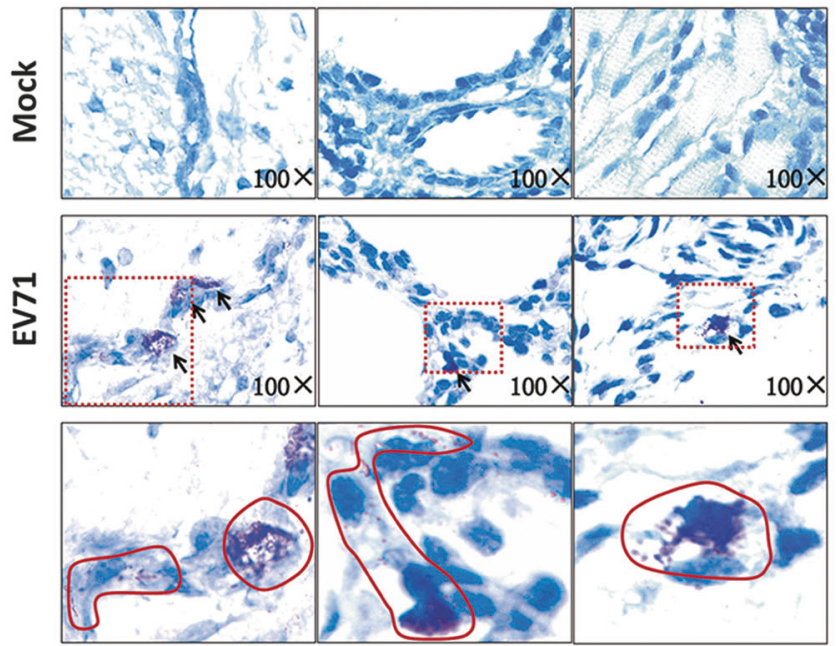

C
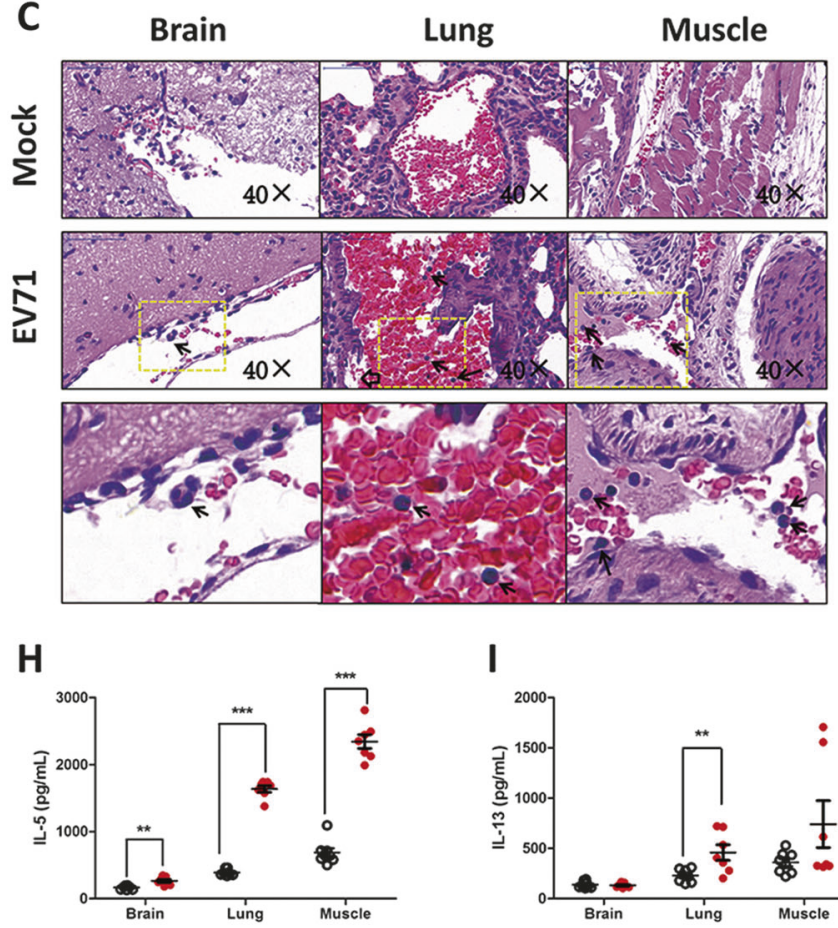

I

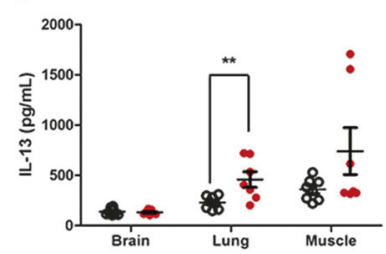

B

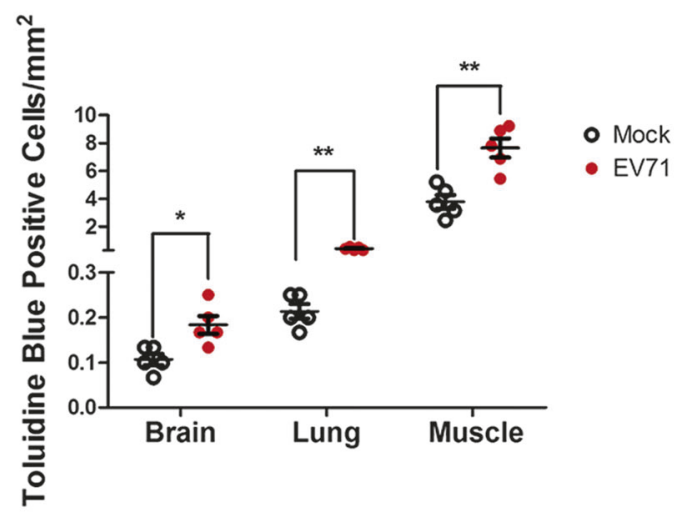

D

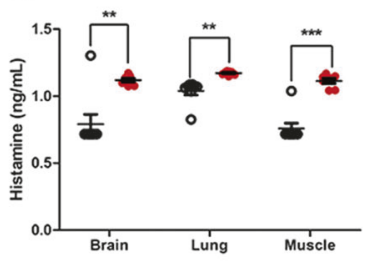

E

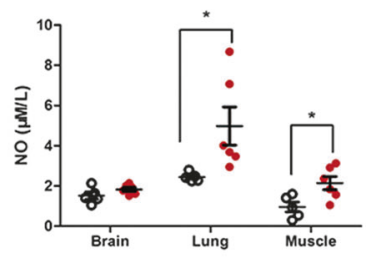

$\mathbf{F}$

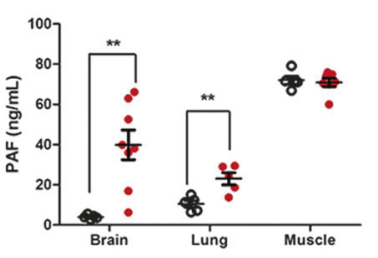

G

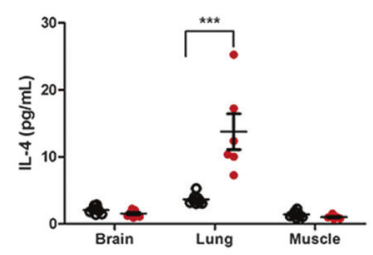

Fig. 3 EV71 infection activated mast cells and induced allergic inflammation in target organs or tissues. Toluidine blue staining was used to observe mast cells in brains, lungs and skeletal muscle of mice after EV71 infection. a Toluidine blue stained brain, lung and skeletal muscle slices of mice with mock and EV71 infection with amplification $(\times 40)$. b Number of toluidine blue-positive cells (mast cells, indicated by black arrows) in brain, lung and skeletal muscle slices of mice with mock $(n=5)$ and EV71 infection $(n=5)$. H\&E staining

inflammatory cytokines that might be derived from different lung immune cells. Eotaxin-1, eotaxin-2, ICAM-1 and IL13 are always associated with allergic inflammation in the presence of mast cell. Next, we detected tissue-resident MCs after EV71 infection.

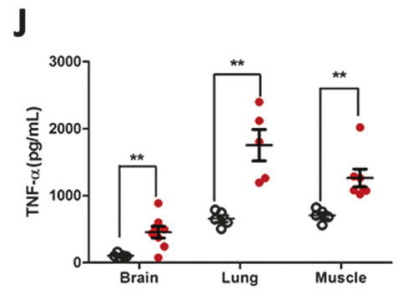

was used to observe eosinphils in brains, lungs and skeletal muscle of mice after EV71 infection. c Eosinphils (indicated by black arrows) in brain, lung and skeletal muscle slices of mice. Levels of histamine (d), NO (e), PAF (f), IL-4 (g), IL-5 (h), IL-13 (i), TNF- $\alpha$ (j) in brain, lung and skeletal muscle lysates from mice with mock $(n=5)$ and EV71 infection $(n=5-7)$ were detected using ELISA kits. The data are expressed as means \pm SEM. $* P<0.05$, vs. Mock; $* * P<0.01$, vs. Mock; $* * * P<0.001$, vs. Mock

\section{EV71 infection activated MCs and induced allergic inflammation in target organs or tissues}

To examine the possible involvement of MCs during EV71 infection, we quantified the number of MCs in the brains, 
A

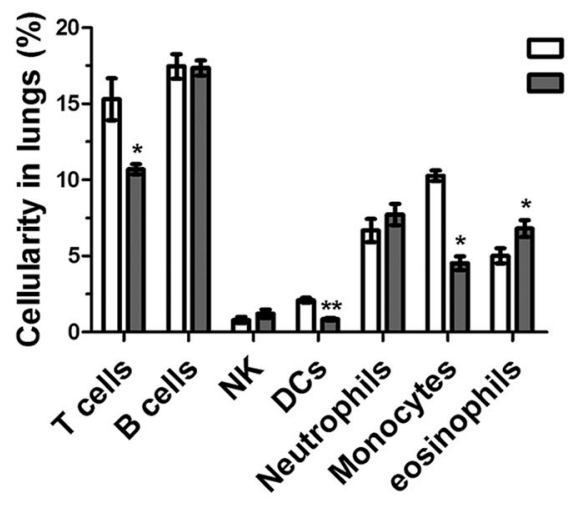

B

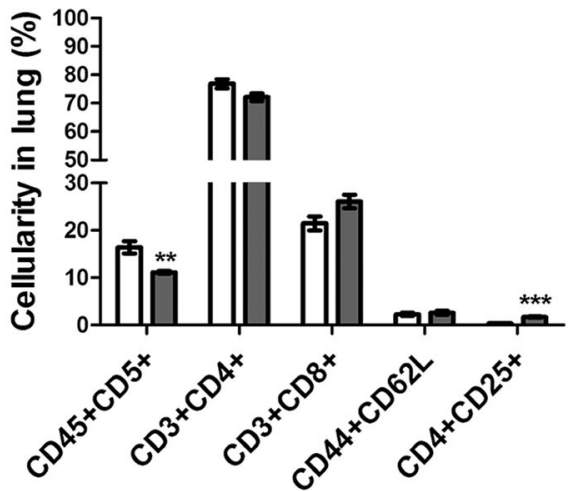

C

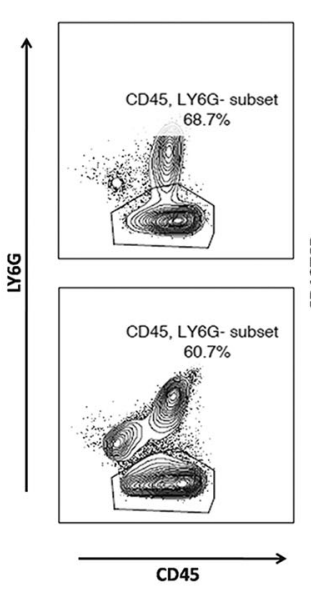

Mock

EV71

EV71 
Fig. 4 EV71 infection increased lung mast cells. To investigate the role of immune cells in the process of EV71 infection, the whole lung immune cells were analyzed by Flow Cytometry. a The proportion of lung immune cells from mock- $(n=4)$ and EV71-infected mice $(n=$ 4). $\mathbf{b}$ The proportion of lung $\mathrm{T}$ cells with different phenotypes in mice with mock $(n=4)$ and EV71 infection $(n=4)$. c CD117 surface expression of mast cells. $\mathbf{d}$ The proportion of lung mast cells in mice with mock $(n=4)$ and EV71 infection $(n=4)$. e Expression of Foxp3 in lung $\mathrm{T}$ cells. $\mathbf{f}$ Mean fluorescence intensity (MFI) of Foxp3 in lung T cells of mice with mock $(n=7)$ and EV71 infection $(n=10)$. $\mathbf{f}$ FACs analysis of lung DCs. The data are expressed as means \pm SEM. $* P<0.05$, vs. Mock; $* * P<0.01$, vs. Mock; $* * * P<0.001$, vs. Mock

infection (Fig. 3b). MCs are known to release granules to recruit inflammatory cells such as eosinophils and cause increased vascular permeability. We stained the brains, lungs and skeletal muscle of mice to identify eosinophils; eosinophils cluster in the brains, lungs and skeletal muscles of mice was observed in Fig. 3c. By degranulation, mast cell can release a variety of highly active mediators, including histamine, NO, PAF and 5-HT. The histamine level in the brains, lungs and skeletal muscle of the infected mice was significantly enhanced (Fig. 3d), NO production in the lungs and skeletal muscle of the infected mice was also increased (Fig. 3e), and PAF level was elevated in the brains and lungs of the infected mice (Fig. 3f). Our results also showed that, after EV71 infection, striking increases were observed in IL- 4 and TNF- $\alpha$ expression level in lungs (Fig. 3g-J), IL-5 expression level in brains (Fig. 3h), lungs and skeletal muscle, IL-13 expression level in lungs and skeletal muscle of mice(Fig. 3i). Overall, EV71 infection could cause MCs accumulation, activation and allergic inflammation in brain, lung and skeletal muscle.

\section{EV71 infection increased lung mast cells}

To detect changes of lung immune cells in during EV71 infection-induced edema, the single cell suspension from lung was analyzed by Flow Cytometry. As shown in Fig. 4a, the percentages of $\mathrm{T}$ cells, DCs and monocytes were decreased after EV71 infection, while the percentages of lung eosinophils was increased. There were no significant differences in the percentages of NK cells, B cells and neutrophils. Because the percentage of $\mathrm{T}$ cells was decreased, we further analyzed which $\mathrm{T}$ cells subsets was reduced. As shown in Fig. 4b, there was no change in CD4 + and CD8 $+\mathrm{T}$ cells after EV71 infection, while the percentage of Treg (CD4+ CD25+) was increased in EV71infected lungs. Additionally, the percentage of CD4+ Foxp3 $+\mathrm{T}$ cells (Fig. 4e) and mean fluorescence intensity (MFI) of Foxp3 (Fig. 4f) were also increased after EV71 infection. In agreement with our data in Fig. 3, the number of MCs (Fig. 4c, d) was increased in EV71-infected lungs. These results indicate that EV71 infection reduced the numbers of T cells, DCs and monocytes population, while increasing those of eosinophils, Treg and MCs in the lung.

\section{MCs participated in the pathogenesis of EV71 infection-induced pulmonary edema}

To verify whether MCs contribute to severe symptoms induced by EV71 infection, sixteen 3-day-old neonatal mice were i.p. inoculated with ZZ1350 strain $\left(2 \times 10^{6} \mathrm{PFU}\right)$ or culture supernatants. EV71-infected mice, based on clinical scores at $7 \mathrm{dpi}$, were further divided into mild and severe subsets.

Mean clinical scores of mice at 7 dpi were showed in Fig. 5a. As shown in Fig. 5b, representative images of congestive lungs were observed in mice with severe symptoms, and the ratio of wet lung weight/ body weight in mice with symptoms was significantly increased. Supplementary Figure S1 showed that EV71 infection caused increased degree of CNS lesions in mice with mild and severe symptoms. As shown in Fig. 5c, h, e, Masson and PAS stained lung slices showed that EV71 infection induced more severe vascular leakage and mucus production in mice with severe symptoms, which were supported by pathological scores. Additionally, viral VP1 expression (Fig. 5d) and viral titers (Fig. 5e) in lung tissues were increased in mice with severe symptoms compared to mild mice.

Next, we examined whether MCs facilitated the pathogenesis of EV71 infection. Toluidine blue staining and immunofluorescence were used to measure MCs number and tryptase expression, an enzyme released from MCs, which were shown in Figure S2 and Fig. 5f, separately. Compared to mild and control mice, severe mice exhibited not only significant-increases in MCs number (Fig. 5g) and tryptase expression (Fig. 5h), but also a trend towards an increase from control to severe mice. Furthermore, there were positive correlations between MCs number in brain $(r$ $=0.701, P=0.003)$, lung $(r=0.802, P<0.0001)$, skeletal muscle $(r=0.737, P=0.001)$ and mean clinical score, as shown in Fig. 5i-k. Thus, our results appears that MCs might be involved in the pathogenesis of EV71 infectioninduced severe symptoms with pulmonary edema.

\section{Discussion}

Millions of infants and young children still suffer from HFMD globally every year [2, 24]. Asymptomatic, acute, severe, and even persistent infections in patients have been documented. Among HFMD patients, an alarming feature was the fatal pulmonary edema associated with severe brainstem encephalitis [4, 25]. However, the potential mechanisms underlying EV71 infection-induced pulmonary 

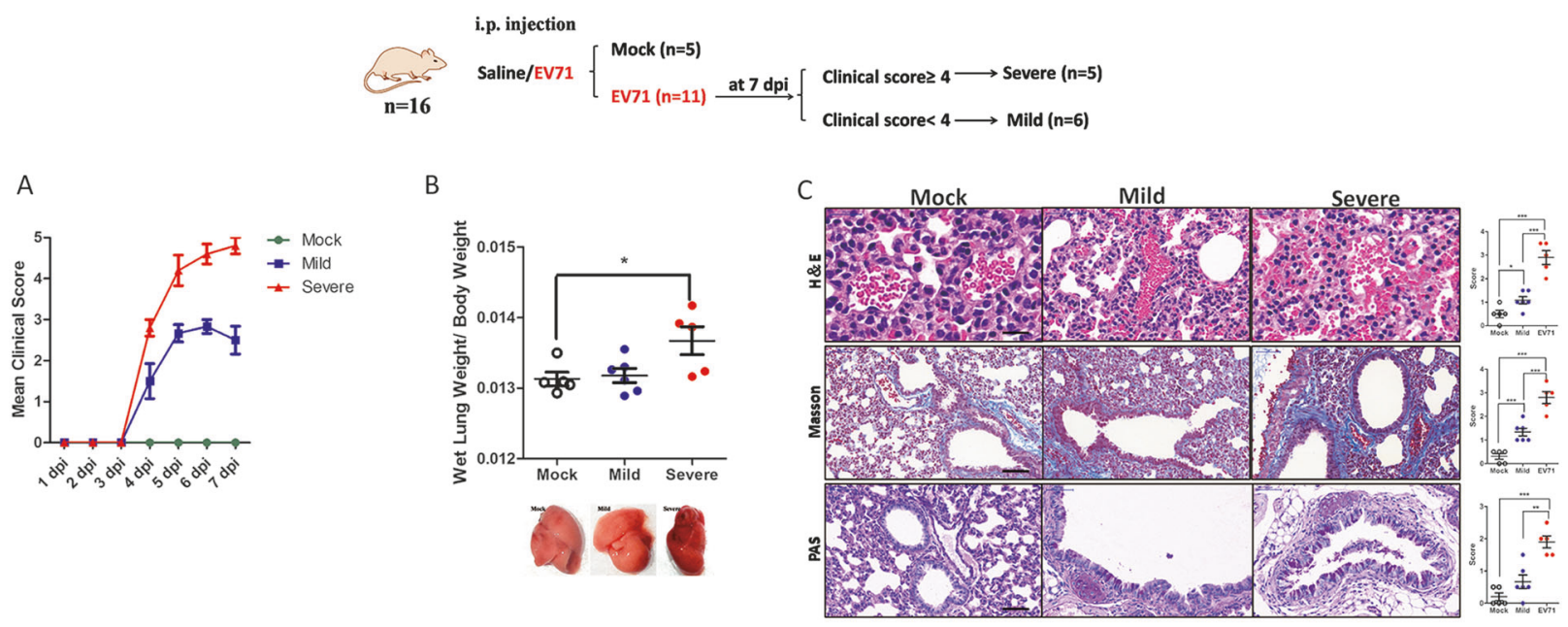
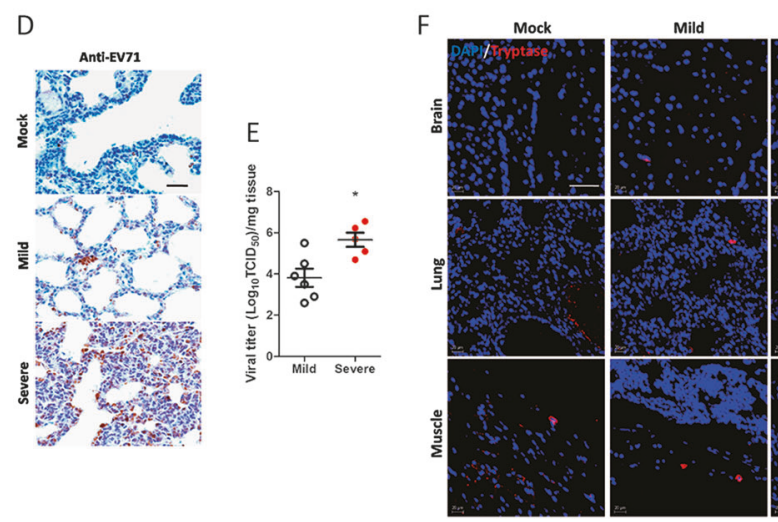

Fig. 5 Mast cells participated in EV71 infection-induced pulmonary edema. Sixteen 3-day-old neonatal mice were i.p. inoculated with ZZ1350 strain $\left(2 \times 10^{6} \mathrm{PFU}\right)$ or same volume of RD cell culture supernatants, and then were divided into mild and severe symptoms with clinical score at 7 dpi. a Mean clinical score of control mice $(n=$ $5)$ and mice with mild $(n=6)$ or severe symptoms $(n=5)$. b Ratio of wet lung weight/body weight of mice and representative image of mice lungs. c H\&E, Masson's Trichrome and PAS stained lung slices, which were supported by histology score. d IHC staining of EV71 VP1 expression in mice lungs. $\mathbf{e}$ Viral titters of lungs in EV71-infected mice with mild $(n=6)$ or severe symptoms $(n=5)$. Toluidine blue

edema have been underexplored. The proliferation of mast cells have been found to be associated with various pathogens $[9,10]$. Herein, we established a mouse model that was infected by EV71 with CNS lesions and pulmonary disorders, and analyzed the role of MCs in the process of EV71 infection. Our results showed that EV71 infection caused CNS lesions and pulmonary edema in neonatal mice, and the number of MCs number in brain, lung and skeletal muscle of mice was increased and correlated with the severity of clinical symptoms after EV71 infection.

As described in our previous study [17], EV71 strain (C4 subtype) used in the present study was isolated from a nonfatal case with CNS involvement. Our results also suggested that the EV71 strain is high cytotoxic and could replicate rapidly in vitro. One and 3-day-old BALB/c mice were highly susceptible to this non-mouse-adapted EV71 strain and exhibited various repertoire of clinical symptoms ranging from skin lesions (data not shown) to paralysis, ataxia, tremors, acute encephalomyelitis and even respiratory disorders. Young immunodeficient AG129 mice were previously reported to display polio-like neuropathogenesis upon infection with a non-mouse-adapted EV71 strain [26]. Compared with this AG129 mice, our animal model might be advantageous in terms of its similarity to HFMD development in infants and young children. Because of high mortality of 1-day-old BALB/c mice after EV71 infection, 3-day-old mouse model with high incidence of neuropathological features was chosen for further study, which was close to clinical complications of HFMD. On the basis of the histopathological analysis at the time of 
Fig. 6 Proposed mechanism of action for mast cell in the the pathogenesis of EV71 infection

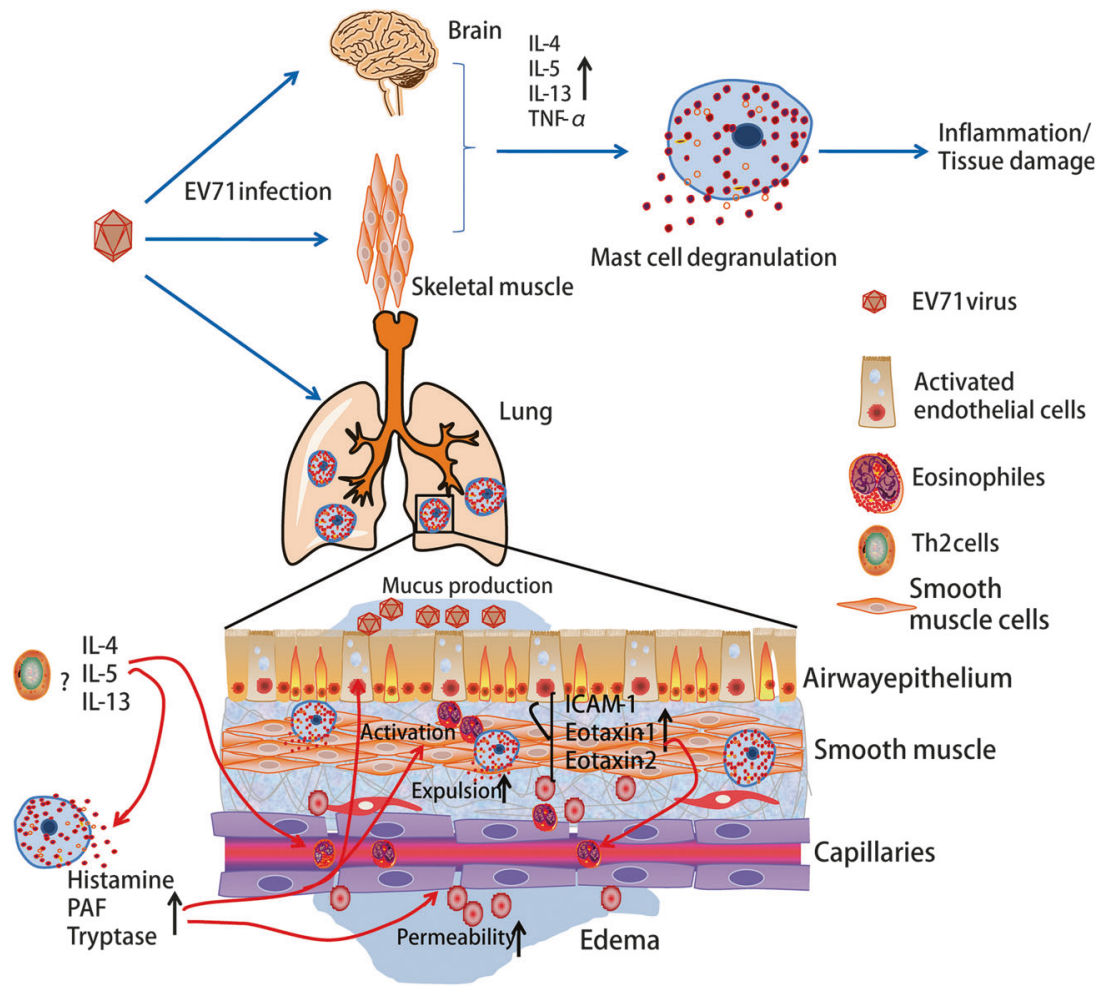

killing, the brains, spinal cords and skeletal muscle of infected mice exhibited different severity of lesions and viral antigens. These results were consistent with the previous reports that EV71 virus mainly invaded CNS and muscle $[19,26]$.

Pulmonary edema is mainly responsible for fatal HFMD [3, 4], which could be defined as an extra-vascular increasing fluid in the lungs [27]. $L_{\mathrm{m}}$, EG-VEGF and NA are known as the indicators of pulmonary edema [28-30]. In our study, we found increased lung mass, $L_{\mathrm{m}}$, EG-VEGF and NA in lung lysates, mucus production and erythrocytefilled fluid in the alveolar spaces of mice lungs after infection. These features were similar to previous reports involving EV71 infection-induced pulmonary edema in vivo and human fatal cases [29, 31, 32]. Interestingly, inflammatory cytokines such as eotaxin-1, eotaxin-2, ICAM-1, IL-7, IL-13 and IL-17 were clearly elevated in lung lysates after EV71 infection, while G-CSF, IFN- $\gamma$ and IL-10 were drastically decreased. Eotaxin- 1 and eotaxin-2 can be secreted by activated endothelial cells, mast cell or eosinophil, and are known to cause $\mathrm{Th} 2$ allergic reaction by recruiting eosinophils [33, 34]. MCs can modulate adhesion molecules on endothelial cells. Activated endothelial cells express ICAM-1 $[35,36]$. IL-13 is known as an important cytokines produced by Th2 cells. It can lead to Th2 allergic inflammation accompanied by mast cell involvement [10]. Therefore, we speculated that MCs might be associated with the pathogenesis of EV71 infection.
Many publications have revealed that MCs play a critical role in the defense against pathogens $[9,10]$. In the current study, we found degranulated MCs in the slices of brain, lung and skeletal muscle of infected mice. The number of MCs were also increased in these tissues. In addition, histamine, NO, PAF were elevated in lung lysates and partially in brain or skeletal muscle. These bioactive chemicals can be released by MCs through degranulation [13, 37]. These evidences further supported mast cell activation during EV71 infection. Th2 cytokines such as IL-4, IL-5 and IL-13 and TNF- $\alpha$ were increased in lung lysates and partially in brain or skeletal muscle. We speculated that EV71 infection caused allergic inflammation especially in lungs of mice. Th2 responses are often associated with increased numbers of tissue-type MCs and eosinophils infiltration during pathogens' infection [10, 13], which was consistent with our results. It has been reported that, during infection and allergic responses, IL-4 and IL-13 act on the epithelium to increase mucosal permeability and decrease glucose absorption and chloride secretion. By contrast, IL-4 enhances mast cell-dependent histamine responses [11]. Mast cell degranulation and Th2 cytokines release were known to cause eosinophils infiltration and further lead to vascular leakage and edema [38], which was observed in our mice after infection. Therefore, we speculated that EV71 infection induced chronic allergic inflammation, which was involved with MCs, especially in lung section. For further study, we analyzed lung immune cells during 
EV71 infection. We found that EV71 infection significantly reduced the numbers of $\mathrm{T}$ cells, DCs and monocytes populations in the lung, while mast cells, eosinophils populations were significantly increased during EV71 infection. These results could explain why IFN- $\gamma$, G-CSF in lung lysates were decreased after EV71 infection. Foxp3 + Tregs play a critical role in immune tolerance and control of Th2 immune responses [39]. In our study, we found that Foxp3+ Tregs were increased in mice lungs during EV71 infection. Overall, our results suggested chronic allergic inflammation-induced by EV71 infection might be associated with pulmonary edema.

Next, we found that MCs were involved in EV71 infection-induced pathogenesis, especially for lung disorders. It was reported that influenza infection in mice induced accumulation of lung MCs, which might be associated with exacerbations of lung disorders [22]. In the present study, we found that MCs number in brain, lung and skeletal muscle posed a positive correlation with the severity of symptoms-induced by EV71 infection, especially for lung disorders. Severe mice with pulmonary edema exhibited higher viral titters in lung tissue, which was also found in previous study [32]. The release of histamine and other vasoactive mediators from mast cell can increase vascular permeability and local blood flow, and act on smooth muscle to increase the expulsion of mucosal parasites $[9,10,13]$. In addition, it was reviewed that histamine could induce epithelial cell mucus production, which might aid in pathogen immobilization and cytoprotection [9, 10]. Mast cell production of chemotactic factors can enhance the recruitment of multiple inflammatory cells, including eosinophils (eotaxin), NK cells, neutrophiles and CD8 + T cells $[9,10,40]$. In our study, we found increased numbers of eosinophils (eotaxin), NK T cells, neutrophils and $\mathrm{CD} 8+\mathrm{T}$ cells in the lung. The CD8 $+\mathrm{T}$ cells in blood were decreased, while the activated $\mathrm{CD} 8+\mathrm{T}$ cells were increased after EV71 infection (Supplementary Figure S3). The above evidences indicated that EV71 infection caused mast cell degranulation in the sites of infection, and subsequent overproduction of proinflammatory mediators, which might further lead to host tissues damage and severe symptoms, especially for lung section.

In conclusion, our present study for the first time found that mast cell might contribute to the pulmonary edema during EV71 infection (Fig. 6). In our proposed model, specific inhibitors of mast cell degranulation could be beneficial as therapy to treat EV71 infection-induced severe HFMD with pulmonary edema.

Acknowledgements We thank Yinming Liang of Laboratory of Genetic Regulators in the Immune System, School of Laboratory Medicine, Xinxiang Medical University for helping us perform Flow Cytometry analysis. We thank Po-Hsun Huang of Department of Mechanical Engineering and Materials Science, Duke University for article's edit. This work was funded by the National Natural Science Foundation of China (81172740); National Natural Science Foundation of China (81573205); Key scientific research projects in Colleges and Universities of Henan Province (15A330003); Outstanding doctoral thesis training fund of Zhengzhou University.

\section{Compliance with ethical standards}

Conflict of interest The authors declare that they have no conflict of interest.

\section{References}

1. Duan G, Yang H, Shi L, et al. Serum inflammatory cytokine levels correlate with hand-foot-mouth disease severity: a nested serial case-control study. PLoS ONE. 2014;9:e112676.

2. Solomon T, Lewthwaite P, Perera D, et al. Virology, epidemiology, pathogenesis, and control of enterovirus 71. Lancet Infect Dis. 2010;10:778-90.

3. Ho M, Chen ER, Hsu KH, et al. An epidemic of enterovirus 71 infection in Taiwan. Taiwan Enterovirus Epidemic Working Group. N Engl J Med. 1999;341:929-35.

4. Lum LC, Wong KT, Lam SK, et al. Neurogenic pulmonary oedema and enterovirus 71 encephalomyelitis. Lancet. 1998;352:1391.

5. Ooi MH, Wong SC, Lewthwaite $\mathrm{P}$, et al. Clinical features, diagnosis, and management of enterovirus 71. Lancet Neurol. 2010;9:1097-105.

6. Rivera A, Siracusa MC, Yap GS, et al. Innate cell communication kick-starts pathogen-specific immunity. Nat Immunol. 2016; 17:356-63.

7. Pathinayake PS, Hsu AC, Wark PA. Innate Immunity and Immune Evasion by Enterovirus 71. Viruses. 2015;7:6613-30.

8. Liu Y, Zhu M, Nishida K, et al. An essential role for RasGRP1 in mast cell function and IgE-mediated allergic response. J Exp Med. 2007;204:93-103.

9. Urb M, Sheppard DC. The role of mast cells in the defence against pathogens. PLoS Pathog. 2012;8:e1002619.

10. Abraham SN, St, John AL. Mast cell-orchestrated immunity to pathogens. Nat Rev Immunol. 2010;10:440-52.

11. McLeod JJ, Baker B, Ryan JJ. Mast cell production and response to IL-4 and IL-13. Cytokine. 2015;75:57-61.

12. Wilgus TA, Wulff BC. The importance of mast cells in dermal scarring. Adv Wound Care. 2014;3:356-65.

13. Amin $\mathrm{K}$. The role of mast cells in allergic inflammation. Respir Med. 2012;106:9-14.

14. Yamaya M. Virus infection-induced bronchial asthma exacerbation. Pulm Med. 2012;2012:834826.

15. Smith-Norowitz TA, Carvajal-Raga S, Weedon J, et al. Increased seroprevalence of Enterovirus $71 \mathrm{IgE}$ antibodies in asthmatic compared with non-asthmatic children. Ir J Med Sci. 2017; 186:495-503.

16. Lee ZM, Huang YH, Ho SC, et al. Correlation of symptomatic enterovirus infection and later risk of allergic diseases via a population-based cohort study. Medicine (Baltim). 2017;96:e5827.

17. Dang D, Zhang $C$, Zhang R, et al. Involvement of inducible nitric oxide synthase and mitochondrial dysfunction in the pathogenesis of enterovirus 71 infection. Oncotarget. 2017;8:81014-26.

18. Liu CC, Lian WC, Butler M, et al. High immunogenic enterovirus 71 strain and its production using serum-free microcarrier Vero cell culture. Vaccine. 2007;25:19-24.

19. Wang YF, Chou CT, Lei HY, et al. A mouse-adapted enterovirus 71 strain causes neurological disease in mice after oral infection. $\mathrm{J}$ Virol. 2004;78:7916-24. 
20. Thurlbeck WM. Internal surface area and other measurements in emphysema. Thorax. 1967;22:483-96.

21. Mizutani N, Nabe T, Yoshino S. Complement C3a regulates late asthmatic response and airway hyperresponsiveness in mice. $\mathrm{J}$ Immunol. 2009;183:4039-46.

22. Zarnegar B, Mendez-Enriquez E, Westin A, et al. Influenza infection in mice induces accumulation of lung mast cells through the recruitment and maturation of mast cell progenitors. Front Immunol. 2017;8:310.

23. Li H, Li Q, Du X, et al. Lithium-mediated long-term neuroprotection in neonatal rat hypoxia-ischemia is associated with antiinflammatory effects and enhanced proliferation and survival of neural stem/progenitor cells. J Cereb Blood Flow Metab. 2011;31:2106-15.

24. Huang PN, Shih SR. Update on enterovirus 71 infection. Curr Opin Virol. 2014;5:98-104.

25. Chang LY, Huang YC, Lin TY. Fulminant neurogenic pulmonary oedema with hand, foot, and mouth disease. Lancet. 1998;352:367-8.

26. Khong WX, Yan B, Yeo H, et al. A non-mouse-adapted enterovirus 71 (EV71) strain exhibits neurotropism, causing neurological manifestations in a novel mouse model of EV71 infection. $\mathbf{J}$ Virol. 2012;86:2121-31.

27. Robin ED, Cross CE, Zelis R. Pulmonary edema. New Engl J Med. 1973;288:292-304.

28. Feng F, Jin Y, Duan L, et al. Regulation of ozone-induced lung inflammation by the epidermal growth factor receptor in mice. Environ Toxicol. 2015;31:2016-27.

29. Victorio $\mathrm{CB}, \mathrm{Xu} \mathrm{Y}, \mathrm{Ng} \mathrm{Q}$, et al. A clinically authentic mouse model of enterovirus 71 (EV-A71)-induced neurogenic pulmonary oedema. Sci Rep. 2016;6:28876.

30. Sato T, Paquet-Fifield S, Harris NC, et al. VEGF-D promotes pulmonary oedema in hyperoxic acute lung injury. J Pathol. 2016;239:152-61.
31. Rogers FB, Shackford SR, Trevisani GT, et al. Neurogenic pulmonary edema in fatal and nonfatal head injuries. J Trauma. 1995;39:860-6. discussion866-868

32. Xu F, Yao PP, Xia Y, et al. Enterovirus 71 infection causes severe pulmonary lesions in gerbils, meriones unguiculatus, which can be prevented by passive immunization with specific antisera. PLoS ONE. 2015;10:e0119173.

33. Menzies-Gow A, Ying S, Phipps S, et al. Interactions between eotaxin, histamine and mast cells in early microvascular events associated with eosinophil recruitment to the site of allergic skin reactions in humans. Clin Exp Allergy. 2004;34:1276-82.

34. Conroy DM, Williams TJ. Eotaxin and the attraction of eosinophils to the asthmatic lung. Respir Res. 2001;2:150-6.

35. Bacon AS, McGill JI, Anderson DF, et al. Adhesion molecules and relationship to leukocyte levels in allergic eye disease. Invest Ophthalmol Vis Sci. 1998;39:322-30.

36. Gudbjornsson B, Hallgren R, Nettelbladt O, et al. Phenotypic and functional activation of alveolar macrophages, $\mathrm{T}$ lymphocytes and NK cells in patients with systemic sclerosis and primary Sjogren's syndrome. Ann Rheum Dis. 1994;53:574-9.

37. Reber LL, Hernandez JD, Galli SJ. The pathophysiology of anaphylaxis. J Allergy Clin Immunol. 2017;140:335-48.

38. Theoharides TC, Alysandratos KD, Angelidou A, et al. Mast cells and inflammation. Biochim Biophys Acta. 2012;1822: 21-33.

39. Venuprasad K, Kong YC, Farrar MA. Control of Th2-mediated inflammation by regulatory $\mathrm{T}$ cells. Am J Pathol. 2010; 177:525-31.

40. Ebert S, Becker M, Lemmermann NA, et al. Mast cells expedite control of pulmonary murine cytomegalovirus infection by enhancing the recruitment of protective CD8 T cells to the lungs. PLoS Pathog. 2014;10:e1004100. 